\title{
The Impact of Technology on Contemporary Accounting: An ABCD Perspective
}

\author{
Lawrence A. Gordon \\ EY Alumni Professor of Managerial Accounting and Information Assurance at the Robert H. Smith School \\ of Business, University of Maryland, USA
}

\section{Introduction}

We live in an interconnected digital world of rapidly changing technology. This technology has created dynamic changes in nearly every field of study. The field of accounting is no exception in this regard. Four aspects of modern technology are having profound effect on the education, practice and research of contemporary accounting. These aspects of technology are what I call the $\mathbf{A B C D}$ of technology, where $\mathbf{A}$ stands for Artificial Intelligence/Machine Learning, B stands for Blockchain, C stands for Cybersecurity, and D stands for Data Analytics.

There are two major objectives of this paper. The first objective is to briefly discuss the impact of the $A B C D$ aspects of technology on contemporary accounting. The approach to pursuing this objective will be to initially provide a definition of each of these aspects of technology, followed by a discussion on how each aspect of technology is transforming contemporary accounting. This objective is addressed in the next section of the paper. The second objective of this paper, which is addressed in the third section of this paper, is to discuss how the field of accounting needs to change in order to adapt to the $A B C D$ aspects of technology. The fourth section of the paper provides some concluding comments.

\section{The Impact of The ABCD of Technology on Contemporary Accounting}

Artificial Intelligence/Machine Learning: Artificial Intelligence (AI) is usually considered a sub-field within computer science and is concerned with intelligent behavior by computers. The underlying assumption of $\mathrm{Al}$ is that computers (or more broadly, machines) can learn and subsequently perform tasks that previously were considered to require human intelligence. Hence, machine learning is essentially a subfield of Al.

$\mathrm{Al}$, as a separate field of study, began in the mid-1950s. However, the major advances in Al were not possible until the memory capacity and processing speed of computers reached an adequate level to simulate human reasoning and problem solving tasks. Early advances in Al were often referred to as expert systems because they were simulating structured decisions performed by an expert. Today, Al is applied to all forms of structured and unstructured decisions, including decisions where inductive learning and deductive reasoning are an essential part of the decision process.

Accounting focuses on measuring, recording, summarizing, analyzing, processing, and communicating financial, as well as nonfinancial, information about economic activities of organizations and individuals. Furthermore, accounting information is used in making all types of structured, as well as unstructured, decisions. Thus, an accounting information system is a perfect candidate for embracing machine 
processing of information. Indeed, various forms of adding machines and calculators have been used to assist in carrying out accounting activities for centuries. In addition, computers have been used to carry out many of the routine accounting functions for decades.

Recent advances in Al, especially machine learning aspects of $\mathrm{Al}$, have opened up new vistas for the field of accounting. For example, machine leaning is used to develop models for forecasting revenues and costs. The machine (i.e., computer) "learns" how to revise the forecasting models based on a variety of past experiences and real time relevant events, simulating inductive and deductive reasoning. More specifically, based on past deviations between actual and forecasted revenues and costs, combined with additional information such as market demand forecasts, the computer can revise the forecasting model in a manner that resembles inductive and deductive reasoning by human beings. Of key importance is the fact that the revised forecasting model does not have to be based on predetermined programs or algorithms. Other examples of where machine learning is having a significant impact on accounting are in cost allocation models, profit planning models, fraud detection, and tax planning.

Blockchain: A blockchain is a digital decentralized ledger that consists of blocks of transactions between parties. The information in the blockchain is available, and transparent, to the members of the blockchain on a real time basis. Every block in the blockchain includes a link, based on cryptography, which comes from the previous block. Technically speaking, the link is a cryptographic hash function. In order to change the information in a block, a majority of all parties to transactions in subsequent blocks within the chain need to agree to the change. Thus, a large blockchain is extremely secure, even close to immutable (i.e., unchangeable), in terms of protecting the integrity of the information in the blocks. In addition, the availability of the information in a blockchain is stronger than a traditional centralized ledger system because the blockchain's decentralized ledger stores the information in all the blockchain nodes. A blockchain that is open to the public is referred to as a public blockchain. In contrast, a blockchain among a select set of users (e.g., supply chain partners) is referred to as a private blockchain. Hybrid blockchains (i.e., somewhere between public and private blockchains) also exist. Although, blockchains typically use a form of cryptocurrency, a specific country-based currency (e.g., dollars) could also be used.

Besides providing information to the members of a blockchain on a real time basis, transactions conducted via the blockchain can eliminate intermediaries to the transactions (e.g., financial institutions). Accordingly, blockchains facilitate the efficiency of operations and decisions within and between organizations. Hence, accounting systems and controls designed around a blockchain are more cost efficient, as well as more secure, than those based on traditional accounting ledgers. Auditing of the information in a blockchain is also efficiently and quickly carried out. In addition, blockchains permit the preparation of financial reports and the filing of tax information in a speedy and secure manner. Private blockchains among supply chain partners have particular appeal for accounting related transactions and services.

Cybersecurity: Security of information contained in computers became a serious concern ever since personal computers became common back in the late 1970s and early 1980s. However, the commercialization of the Internet in the mid-1990s made computer security a prominent topic of concern. In today's interconnected digital environment, it is common to refer to computer security as cybersecurity. Cybersecurity is concerned with the protection of information accessed and transmitted over the Internet and other computer networks. The primary objectives of cybersecurity are to protect the confidentiality, integrity, and availability (often referred to as $\mathrm{CIA}$ ) of information. Protecting the 
Lawrence A. Gordon; The Impact of Technology on Contemporary Accounting: An ABCD Perspective. Transactions on Machine Learning and Artificial Intelligence, Volume 6 No 5 October (2018); pp: 10-16

confidentiality of information essentially means protecting private information from those that are not authorized to see or use the information. Protecting the integrity of information essentially means protecting the accuracy, reliability and validity of information. Protecting the availability of information essentially means making sure that information is available to authorized users on a timely basis. Two other objectives of cybersecurity that are often considered a subset of availability are authentication and nonrepudiation. Authentication refers to the notion that information has been made available to an authorized user whose identity has been authenticated. Nonrepudiation refers to the notion that an authorized user's identity cannot be repudiated.

Given the role that computers, the Internet, and smart devices play in today's interconnected digital world, cybersecurity breaches are a fundamental concern to the security of nations, businesses and individuals. Unfortunately, cybersecurity breaches have become common within the last two decades. Although most cybersecurity breaches do not threaten the survival of firms, many breaches do result in large costs associated with detecting and correcting the cause of the breach. In addition, some breaches result in significant costs in terms of their negative effects on a firm's brand image and legal liability. The potential negative effects resulting from cybersecurity breaches have resulted in the creation of a vibrant cybersecurity insurance marketplace as a means of transferring the risks associated with potential cybersecurity breaches.

Contemporary accounting systems are dependent on computers and the use of the Internet. Consequently, cybersecurity has become a fundamental concern for accounting. In fact, cybersecurity is a necessary, although not sufficient, condition of reliable and valid financial reporting and management accounting systems. To see how accounting systems are dependent on cybersecurity, consider the requirement for publicly traded firms to report material weaknesses under Section 404 of the SarbanesOxley Act (SOX) of 2002. According to SOX, firms are required to report to the Securities and Exchange Commission (SEC) any material weaknesses in the reliability of their financial reports. However, firms operating in a computer-based environment (which, of course, means all firms) cannot have reliable financial reports without the presence of cybersecurity. Furthermore, if the reporting systems are not reliable, they lack validity and cannot be verified for accuracy. Accordingly, cybersecurity is a fundamental concern to any contemporary computer-based financial reporting or managerial accounting system. In addition, reliable auditing and tax preparation services are also dependent on secure computer-based information systems (i.e., cybersecurity). In this latter regard, the security of tax filings has become a serious concern to tax preparers, as well as the Internal Revenue Service, within the last two decades.

Cybersecurity not only has an impact on accounting, accounting also impacts cybersecurity. In fact, during the past two decades, accountants have examined several issues of importance to cybersecurity. These issues include: the cost of cybersecurity breaches [1], the amount to invest in cybersecurity activities to prevent cybersecurity breaches [2], and the impact on a firm's value derived from disclosing cybersecurity risks and incidents in 10-K reports filed with the SEC [3]. All three of these issues have been, and continue to be, of particular concern to accounting researchers, as well as practitioners.

Data Analytics: Data analytics involves analyzing and interpreting patterns and trends based on large sets of data. The focus of data analytics is on gleaning insights from large sets of data that are not obvious to the naked eye. Analyzing large sets of data is not a new phenomenon. In fact, analyzing and interpreting large sets of data have been a part of the field of statistics for centuries. During the 1990s, analyzing large 
sets of data via statistical techniques to determine patterns not easily identified by the naked eye became popular under the heading of data mining. However, contemporary computer processing capabilities and visualization software, combined with sophisticated statistical analyses, have opened up new doors for those interested in data analytics. Furthermore, the amount of data available for such analyses and interpretation prior to the commercialization of the Internet paled in comparison to what is currently available to organizations. Indeed, the large amount of data that is currently available and can be stored in computers, statistically analyzed, and presented in sophisticated graphs, tables, figures and reports within seconds, far exceeds what most imagined possible as recently as the turn of the 21st century.

The explosion of data availability resulting from the Internet created a large demand for user friendly software for analyzing, interpreting, and visually presenting vast amounts of data. The field of data analytics was the response to this demand. Data analytics software allows data users to analyze, interpret, and visually present, in terms of easy to understand tables, figures, and reports, large amounts of data via a simple click of a key on the computer. Although the underpinnings of data analytics software are based on sophisticated statistics and complex computer programs, users of such software only need a basic understanding of these underpinnings. In other words, the data analytics software has become user friendly.

Data analytics is transforming the way contemporary accounting is analyzing, interpreting and reporting information. In fact, the technology associated with data analytics is shifting the focus of contemporary accounting from performing functions like gathering and summarizing data to that of analyzing and interpreting large data sets. In managerial accounting, for example, it is possible to create real-time analyses, interpretations and visualizations of budgets and forecast variances. It is also possible to use a variety of data available on the Internet (e.g., macroeconomic forecasts of Gross Domestic Product, Investments, etc.) to enhance forecasting and profit planning models. In addition, it is now possible to audit an entire population, rather than a sample, while at the same time producing a comprehensive visualization of the audit's findings. Reporting of company financial reports are also being drastically changed by the field of data analytics. In fact, one can easily imagine the day when publicly traded corporations will provide a link to the company's financial information on a real time basis, supported by tables, reports, figures and graphs in the same way stock market information are currently available.

Interactions in the ABCD Aspects of Technology and Accounting: The above discussion on the impact of the $A B C D$ aspects of modern technology on contemporary accounting has considered each aspect as being independent of each other. In reality, there are important interrelationships among them. For example, the developments related to data analytics are facilitated by the developments related to machine learning and vice versa. The developments related to data analytics are also facilitating the developments related to blockchain. In addition, cybersecurity affects all three of the other aspects of modern technology discussed in this paper. More to the point, since computer networks and the Internet are at the heart of modern technology, cybersecurity is a necessary, although not sufficient, requirement for artificial intelligence/machine learning, blockchain, and data analytics.

Figure 1 illustrates the interrelationships among the $A B C D$ aspects of technology and their direct impact on contemporary accounting. For illustrative purposes, Figure 1 subdivides accounting into the following categories: financial reporting, managerial accounting, auditing, and taxes. Accounting information systems is shown in Figure 1 as the thread that links all the subcategories of accounting. 


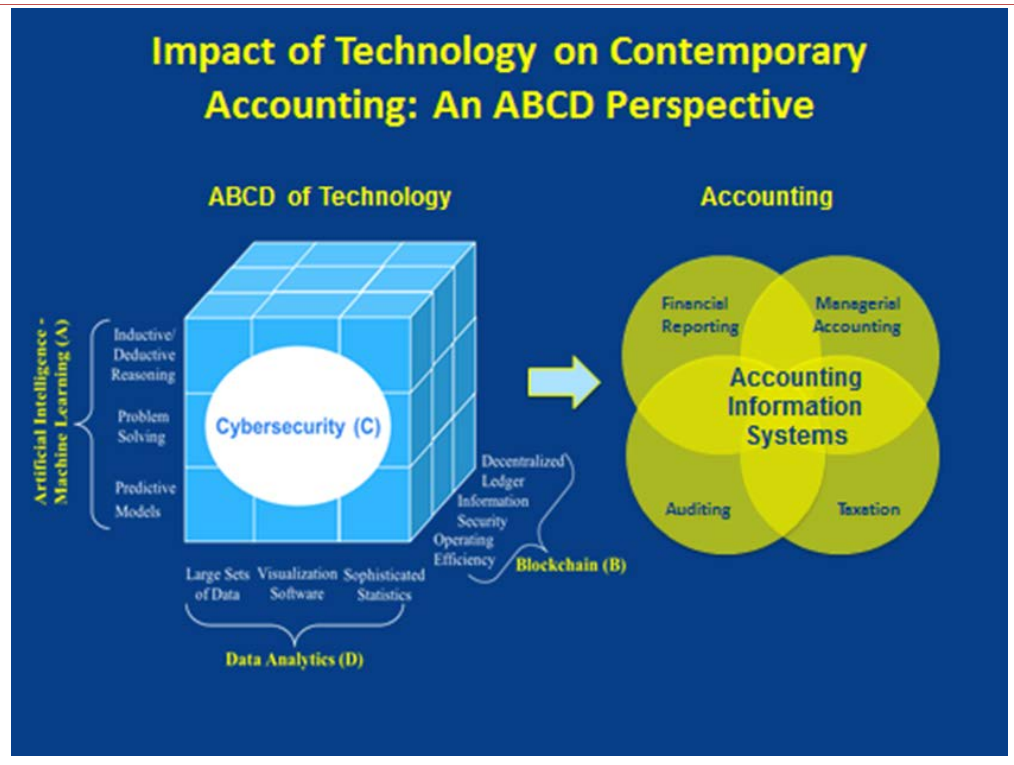

Figure 1

\section{Required Changes}

Accounting educators, practitioners and researchers have all recognized the importance of the $A B C D$ aspects of technology on accounting. However, the implementation of the requisite changes has been slow when compared to the way several other fields are changing in response to the $A B C D$ aspects of technology. As a result, the field of accounting appears to be losing ground compared to other disciplines in terms of attracting the best and brightest individuals. For example, there was a time when majoring in accounting was commonly viewed as the most intellectually challenging and most marketable major for Business School undergraduates. During that time, starting salaries upon graduation were also at the top, or at least near the top, for accounting majors compared to other majors graduating with a bachelor's degree. Much of that has changed in today's technological-based environment. Fields such as business analytics and information systems, which have fully embraced the advances in modern technology, are becoming (and in many cases have become) more prominent majors in many Business Schools. Furthermore, new graduates majoring in these other fields often receive higher starting salaries than new graduates majoring in accounting. A similar phenomenon is occurring in the hiring of rookie faculty members for business schools. Whereas a rookie Ph.D. in accounting once enjoyed the greatest marketability for a new Ph.D. out of a business school, a clear shift has been occurring. New business school faculty in fields that are more fully grounded in technology than accounting are now occupying the limelight.

The above developments notwithstanding, accounting information is still the bloodline of nearly all organizations. However, technological developments have changed, and continue to change, the role and skill requirements of accountants within organizations. Thus, the accounting industry needs to quickly and fully embrace the changes discussed above related to the $A B C D$ aspects of technology. In this regard, accounting educators need to embrace these changes by substantially revising the curriculum requirements for both undergraduate and graduate degrees in accounting. Of course, substantial changes in the educational requirements of accounting programs means that existing, as well as new, accounting faculty need to be prepared to teach courses that are in line with these changes. 
The competitive market for accounting related services provides a powerful economic incentive for accounting practitioners to embrace the $A B C D$ aspects of technology. Thus, it is not surprising that accounting practitioners are currently leading the movement to embrace the integration of the $A B C D$ aspects of technology. However, accounting practitioners need to move more quickly so as to keep up with other fields. Fields like data science and business analytics are more fully, and more quickly, embracing the $A B C D$ aspects of technology in a manner that often incorporates what has previously been thought of as part of the accounting domain (e.g., analyzing and interpreting large sets of financial data derived from financial statements filed with the SEC). If this trend continues, the marketplace may ultimately end up with data science consulting firms performing a large part of the services that were traditionally thought of as accounting related services. To stem this trend, the accounting industry needs to hire individuals who have both the requisite accounting skills and the modern technological skills as part of their formal college education. Many of the master of science degrees in accounting (i.e., MSA degrees) have been designed to produce such graduates, but a large percentage of the top candidates for these programs are entering masters level programs in other fields (e.g., business analytics) due to higher starting salaries for graduate degrees in these other fields. The above is not suggesting that the accounting industry should not be hiring experts from other fields, but rather that the accounting industry needs to provide accounting students who possess the right technological skills, in addition to their accounting knowledge, the economic incentives that are required to attract them to the field of accounting.

Accounting researchers also need to focus on where the field of accounting is heading rather than focusing on the field's past and current activities (i.e., what hockey players refer to as keeping your eye on where the puck is going, not where it is). Starting in the late 1960s, and gaining substantial momentum through the 1980 s, a major paradigm shift took place among accounting researchers. This shift was from examining the impact of accounting related transactions and standards on a firm's accounting income and accounting asset values, to focusing on the impact of accounting related transactions and standards on a firm's market value. This new research paradigm was, and still is, usually referred to as "capital markets research" among accounting scholars. Capital markets research is still the dominant focus in most major accounting Ph.D. programs in the U.S. Given the impact of the $A B C D$ aspects of technology on contemporary accounting, accounting researchers need to recognize that the time has come for a new, or at least modified, dominant accounting research paradigm. For example, the capital markets research paradigm needs to take into consideration the fact that technology is changing the way capital markets operate, the infrastructure underlying capital markets, and how participants in the capital markets interact with each other. Technology is also beginning to change the currency used in capital markets (i.e., recent developments with cryptocurrencies). In essence, technology is changing the very nature of what we call capital markets. Accordingly, a research paradigm that focuses on the interactions among technology and capital markets is needed. This shift in paradigm also needs to incorporate risk management concepts that explicitly consider the transformation that is occurring in society due to rapidly changing technologies. If accounting researchers do not take up this challenge for a new, or at least modified, research paradigm, researchers in related fields will do it for them by incorporating accounting information into their research agenda (a trend that is already occurring).

\section{Concluding Comments}

The interconnected digital world has changed the way nations, organizations and individuals function. These changes are largely the result of technological advances related to computers and the Internet. 
Indeed, the routine activities handled by people in the past, such as collecting and processing information, are efficiently handled by computers today. The same is true for highly structured activities and decisions that utilize internal and external financial information. In contrast, there is a greater need today for individuals who have sophisticated analytical and computer-based skills, especially associated with the $A B C D$ aspects of technology discussed in this paper. Indeed, as a result of the ABCD aspects of technology, all fields are experiencing a fundamental transformation. As with all major technological advances, some fields will thrive in this new environment, while other fields will struggle to survive and even go the way of the horse-and-buggy.

Accounting is at a crossroad in the technological transformation currently taking place. The required changes have been recognized by the accounting industry, but the implementation of these changes is occurring at a slow pace relative to many other business-related fields. Thus, survival of the accounting industry depends on speeding up this technological transformation. The objective of this article has been to argue that artificial intelligence/machine learning, blockchain, cybersecurity, and data analytics are four fundamental aspects of technology that the accounting industry needs to fully embrace, and to do so in a rapid fashion. Of course, there are other aspects of technology that are also important for accounting, many of which the accounting industry has already fully embraced (e.g., social media, mobile devices, and cloud computing).

\section{REFERENCES}

[1] Gordon, L.A., M. P. Loeb, and L. Zhou, "The Impact of Information Security Breaches: Has There Been a Downward Shift in Costs?" Journal of Computer Security, Vol. 19, No. 1, 2011, pp. 33-56.

[2] Gordon, L.A. and M.P. Loeb, "The Economics of Information Security Investment," ACM Transactions on Information and System Security, (November 2002), pp. 438-457.

[3] Gordon, L.A., M.P. Loeb and T. Sohail, "Market Value of Voluntary Disclosures Concerning Information Security," MIS Quarterly, Vol.34, No.3, 2010, pp.567-594. 